\title{
Contrast-induced Encephalopathy after Coil Embolization of an Unruptured Internal Carotid Artery Aneurysm
}

\author{
Yuito Nagamine ${ }^{1}$, Takeshi Hayashi ${ }^{1}$, Yoshiaki Kakehi ${ }^{2}$, Fumitaka Yamane ${ }^{2}$, \\ Shoichiro Ishihara ${ }^{2}$, Akira Uchino ${ }^{3}$ and Norio Tanahashi ${ }^{1}$
}

\begin{abstract}
A 58-year-old woman developed agraphia and mild right hemiparesis approximately one month after undergoing coil embolization of an unruptured left internal carotid artery aneurysm. MRI performed on day 39 post-coil embolization showed multiple lesions in the white matter with signal hyperintensity on T2-weighted and FLAIR images in the left middle cerebral artery territory. The patient's cerebrospinal fluid exhibited an elevated protein level at $46 \mathrm{mg} / \mathrm{dL}$; however, no other findings suggested another underlying disease. Corticosteroids were administered, and, by day 50 post-coil embolization, the clinical findings and abnormal features on MRI had improved. The patient was therefore diagnosed with contrast-induced encephalopathy after coil embolization.
\end{abstract}

Key words: contrast-induced encephalopathy, cerebral aneurysm, coil embolization, posterior reversible encephalopathy syndrome (PRES)

(Intern Med 53: 2133-2138, 2014)

(DOI: 10.2169/internalmedicine.53.2380)

\section{Introduction}

Contrast-induced encephalopathy (CIE) due to the use of contrast agents is a rare complication of angiography. This pathology was first reported in 1970 as transient cortical blindness after coronary angiography (1). The clinical manifestations include cortical blindness, seizures and focal neurological deficits. The incidence of CIE ranges between $0.3 \%$ and $1.0 \%$, although it can reach $4 \%$ when hyperosmolar iodinated contrast agents are used (2). One possible reason for this complication is disruption of the blood-brain barrier. We herein report the case of a patient diagnosed with CIE after coil embolization of an unruptured internal carotid artery (ICA) aneurysm.

\section{Case Report}

A 58-year-old woman was diagnosed on brain magnetic resonance imaging (MRI) with an unruptured ICA aneurysm at another hospital in 2012. Her past and family medical histories were unremarkable. She was referred to the Department of Endovascular Neurosurgery at our hospital for a further assessment. Cerebral angiography revealed an unruptured aneurysm with a maximum diameter of $5 \mathrm{~mm}$ at the left ICA-ophthalmic artery junction (Fig. 1A). Coil embolization of the aneurysm was performed at the end of February 2013. The coiling procedure was carried out under general anesthesia with heparinization. The aneurysm was framed with ED coil 14 standard (Kaneka, Osaka, Japan) and one Target 360 Ultra coil, while three Target Helical Ultra coils (Target, Stryker, USA) were placed into the aneurysm while preserving the ophthalmic artery, resulting in a volume embolization rate of $21.9 \%$. A HyperForm $7 \times 7-\mathrm{mm}$ balloon (Micro Therapeutics, Irvine, USA) was intermittently inflated and deflated at the neck of the aneurysm. In order to avoid cerebral ischemia, we discontinued balloon inflation within five minutes and used half inflation when necessary. Postcoiling angiography showed complete occlusion of the aneurysm. Iohexol (316 mL of Omnipaque 300;

\footnotetext{
${ }^{1}$ Department of Neurology, Saitama Medical University International Medical Center, Japan, ${ }^{2}$ Department of Endovascular Neurosurgery, Saitama Medical University International Medical Center, Japan and ${ }^{3}$ Department of Diagnostic Radiology, Saitama Medical University International Medical Center, Japan

Received for publication December 25, 2013; Accepted for publication February 25, 2014

Correspondence to Dr. Yuito Nagamine, yuiton@saitama-med.ac.jp
} 

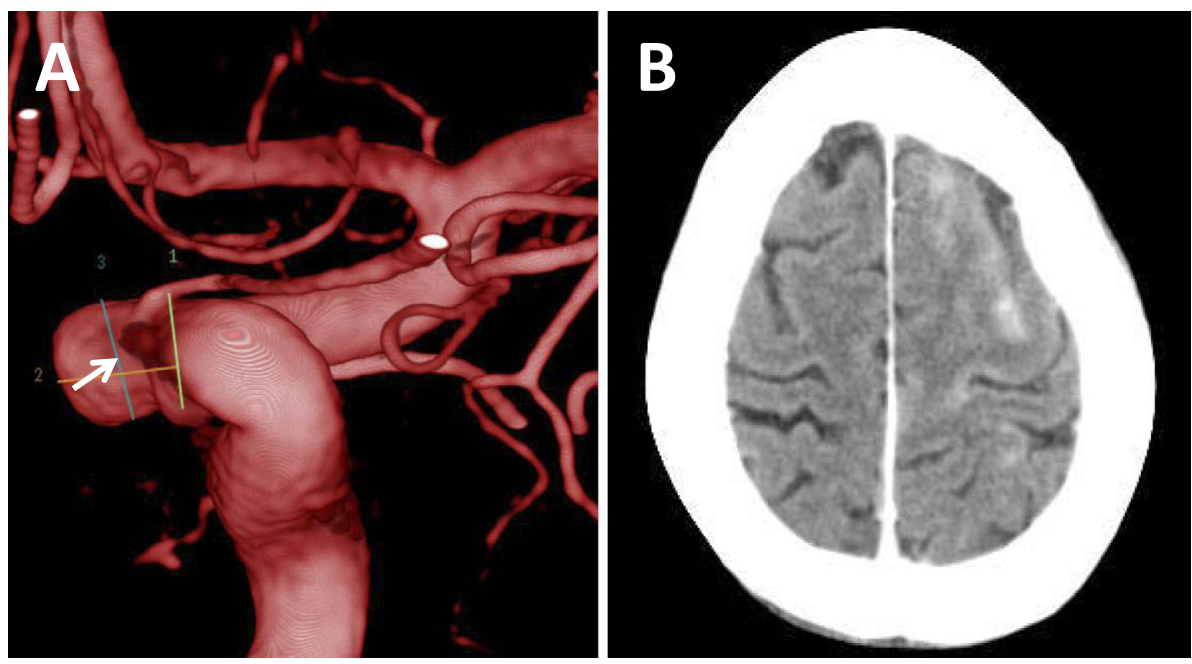

Figure 1. A) Pre-coiling cerebral angiography shows an unruptured aneurysm (arrow) with a maximum diameter of $5 \mathrm{~mm}$ at the branch point of the left ICA-ophthalmic artery. B) Brain CT performed immediately after coiling reveals a hyperdense area due to leakage of contrast agent in the left frontal lobe cortex.

Daiichi Sankyo, Tokyo, Japan) was used as the contrast agent during the procedure. Brain computed tomography (CT) performed immediately after the coiling procedure showed leakage of contrast agent into the left frontal subarachnoid space (Fig. 1B). No large fluctuations in blood pressure were noted and no neurological symptoms were apparent during or after the procedure, while brain MRI performed on day 3 post-coiling revealed only a small, acute infarct in the left insular cortex and high frontal cortex (Fig. 2). The patient was discharged without neurological deficits.

At work, approximately four weeks after hospital discharge, her colleagues pointed out that she was making simple mistakes, such as being unable to write or remember kanji (Japanese characters). Brain MRI performed on day 39 post-coiling showed multiple lesions predominantly in the white matter with signal hyperintensity on T2-weighted and fluid-attenuated inversion recovery (FLAIR) imaging in the territory of the left middle cerebral artery (MCA). In addition, post-contrast MRI revealed punctate enhancement within the lesions (Fig. 3). The patient was therefore readmitted to our hospital for a further evaluation and treatment.

A general physical examination performed on admission disclosed no abnormalities. According to the neurological examination, the patient was lucid, although an assessment of the higher brain function showed agraphia. The Mini Mental State Examination total score was 25, with reductions in the scores for attention and calculation with delayed recall. The patient's visual field and other cranial nerves were normal. Her muscle strength in the right upper extremity was mildly decreased. No sensory deficits, abnormal muscle tonus or involuntary movements were identified. All tendon reflexes in the extremities were normal, without any pathological reflexes. An examination yielded negative results for signs of meningeal irritation.
Laboratory studies showed a normal blood cell count, with a white blood cell count of $4.6 \times 10^{3} / \mu \mathrm{L}$ and an eosinophil count of $1.5 \times 10^{2} / \mu \mathrm{L}(3.3 \%)$. Parameters of the liver and kidney functions, electrolytes and coagulation were all normal. The C-reactive protein level was $0.03 \mathrm{mg} / \mathrm{dL}$. In addition, the patient was negative for antinuclear antibodies, anti-SS-A antibodies, anti-SS-B antibodies, anti-ds-DNA antibodies and anti-neutrophil cytoplasmic antibodies. The soluble interleukin (IL)-2 receptor level was $159 \mathrm{U} / \mathrm{mL}$, which is within the normal limits. A drug lymphocyte stimulation test (DLST) to iohexol yielded negative results. A spinal tap revealed an opening pressure of $150 \mathrm{mmH}_{2} \mathrm{O}$; the cerebrospinal fluid was colorless and clear, with a normal cell count of 15 mononuclear cells $/ \mu \mathrm{L}$, although the protein level was slightly elevated at $46 \mathrm{mg} / \mathrm{dL}$. The immunoglobulin $\mathrm{G}$ index was 0.72 , and the level of myelin basic protein was $<40 \mathrm{pg}$ / $\mathrm{mL}$ (both normal). Testing yielded negative results for oligoclonal bands.

On day 46 post-coiling, with the aim of improving the patient's edema, she was treated with intravenous methylprednisolone at a dose of 1,000 mg/day for three days.

On day 53 post-coiling, fluorodeoxyglucose positron emission tomography showed no areas of abnormal uptake. On day 58 post-coiling, MRI demonstrated a reduction in the size of the lesions, with almost complete resolution by day 90 post-coiling (Fig. 4). The lesions exhibited signal hyperintensity on T2-weighted imaging, mild to low signal intensity on T1-weighted imaging and signal isointensity on diffusion-weighted imaging. The apparent diffusion coefficient was elevated. These findings suggested vasogenic edema.

The mild hemiparesis of the right arm and higher brain dysfunction that had developed approximately four weeks post-coiling improved after hospital admission and were almost completely resolved by day 50 post-coiling. Based on 

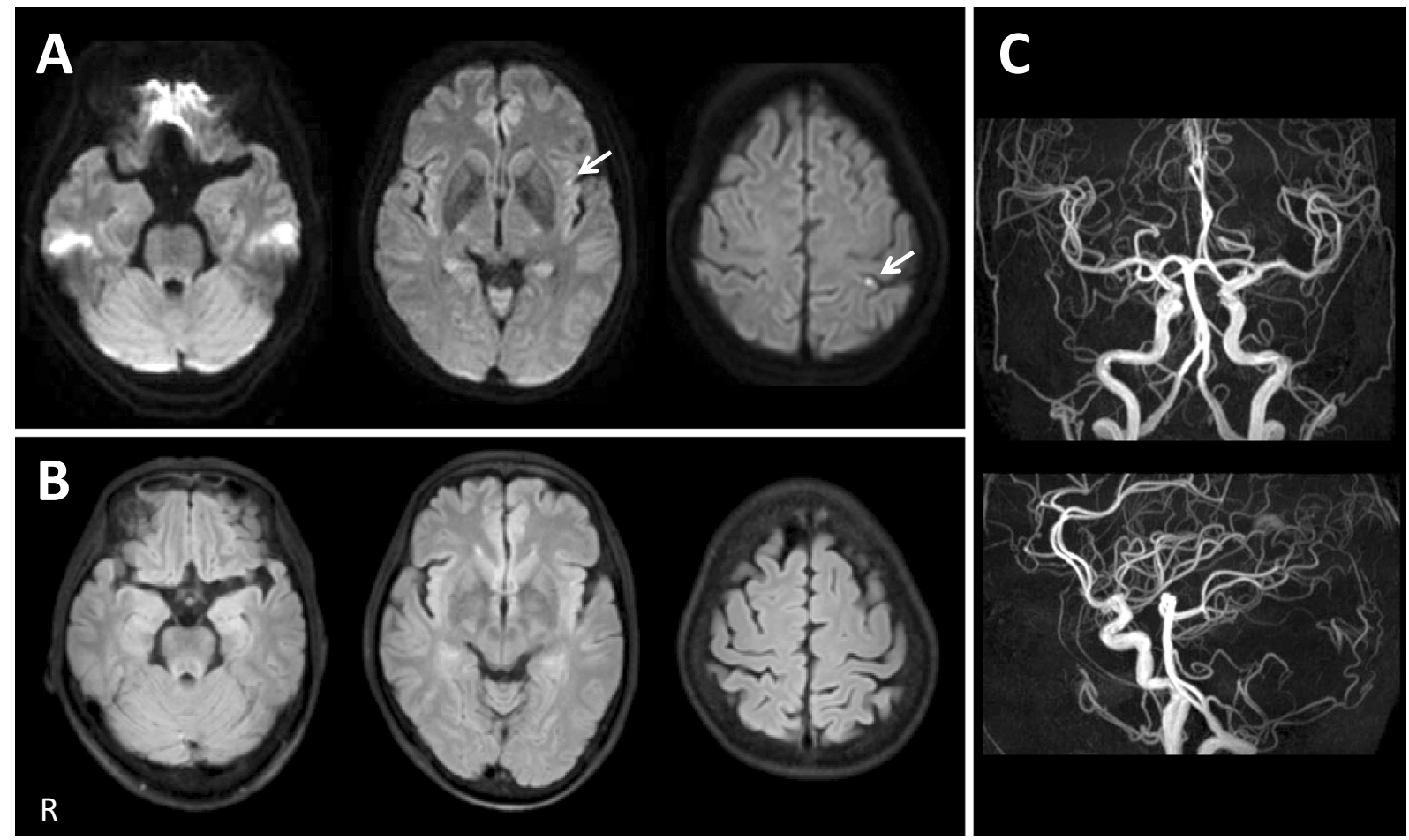

Figure 2. A) Brain MRI performed on day 3 post-coiling shows acute small infarction of the left insular cortex and high frontal cortex on diffusion-weighted imaging (arrows). B) Brain MRI performed on day 3 post-coiling shows no abnormal findings on FLAIR imaging. C) Brain MRA performed on day 3 post-coiling shows no abnormal findings, such us a neck remnant, spasms or occlusion.
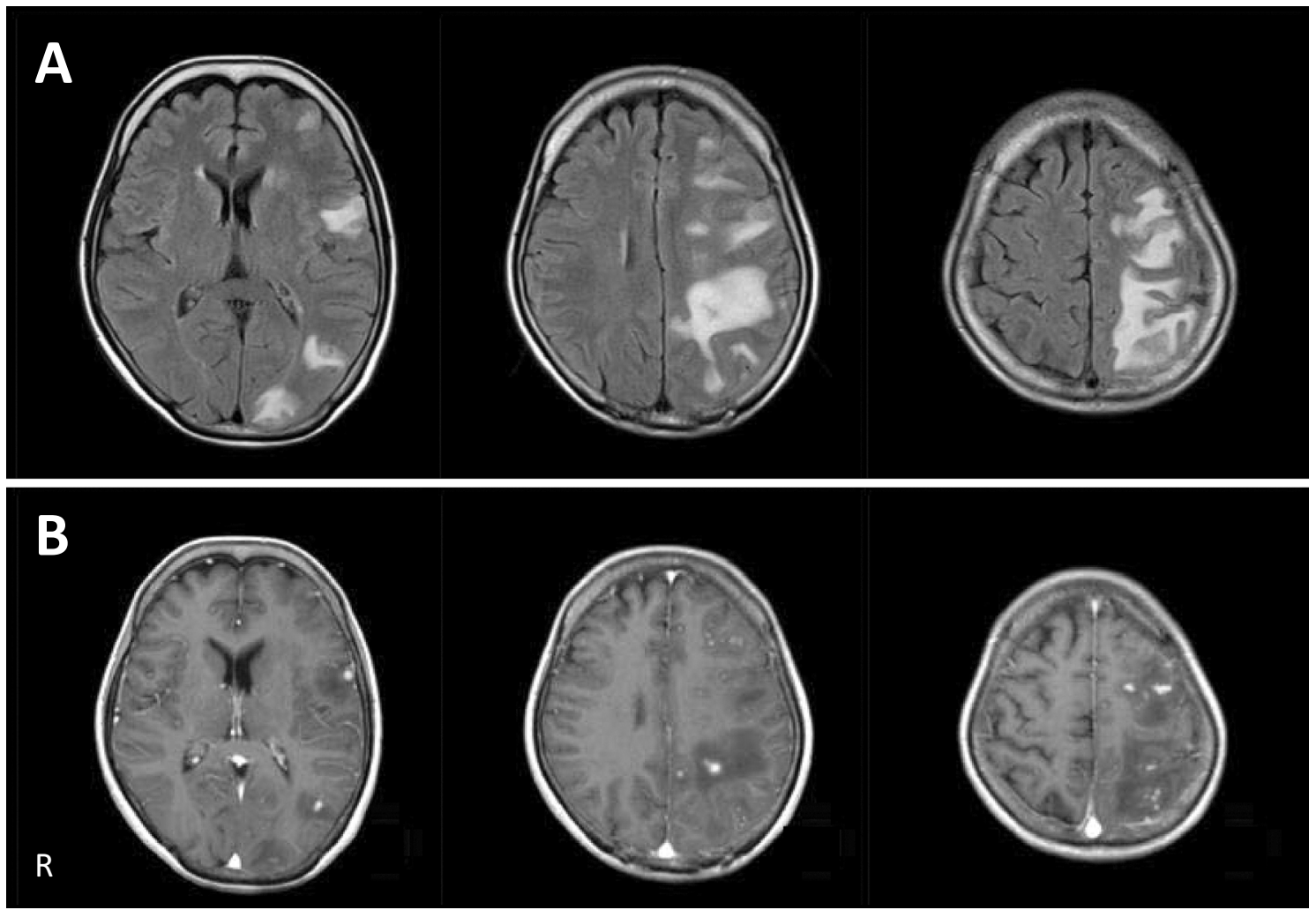

Figure 3. A) Brain MRI performed on hospital admission (39 days post-coiling) shows multiple lesions, predominantly in the white matter, with signal hyperintensity on FLAIR imaging in the area of the MCA in the left cerebral hemisphere. B) Post-contrast MRI reveals punctate enhancement within the lesions. 

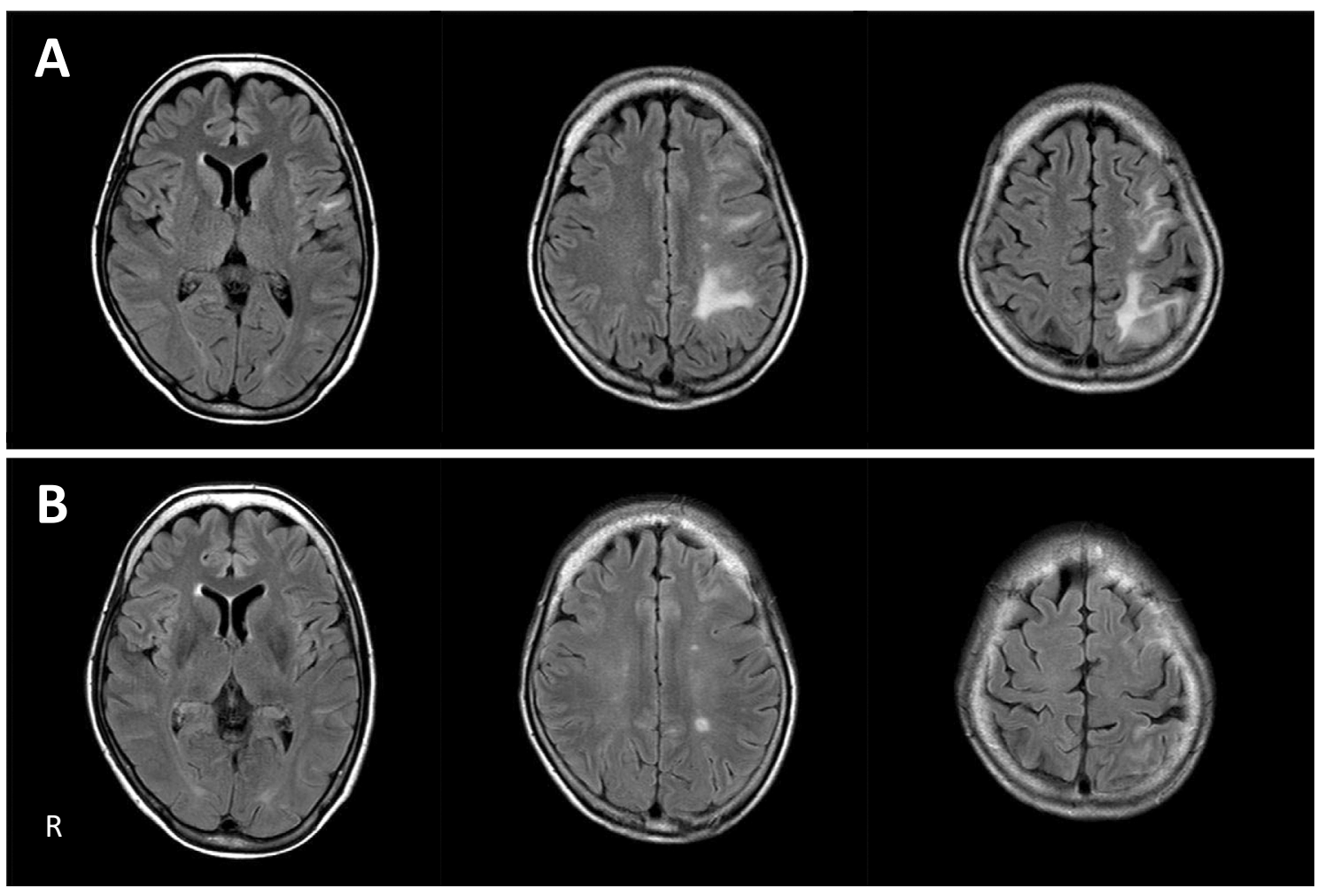

Figure 4. A) Brain MRI performed on day 58 post-coiling shows a reduction in the size of the lesions on FLAIR imaging. B) Brain MRI performed on day 90 post-coiling shows the almost complete resolution of the lesions.

the patient's clinical course and findings on brain MRI, CIE was diagnosed.

\section{Discussion}

The features of the present patient included transient right hemiparesis and agraphia following coil embolization for an unruptured ICA aneurysm, lesions on brain MRI consistent with widespread vasogenic edema predominantly in the white matter territory of the left MCA and the identification of reversible changes over time. Table lists reported cases of CIE after cerebral angiography with CT- or MRI-confirmed lesions (3-18).

Among these cases, including that involving our patient, CIE occurred after coil embolization in 13 of 23 patients. These reports include four cases that developed after coil embolization for ruptured cerebral artery aneurysms $(8,12,14)$. In addition, five cases occurred after cerebral angiography $(3,4,15,17)$, four after carotid artery stenting $(5,10,13)$ and one after placement of a flow diversion stent for an arterial aneurysm (11). Factors associated with CIE include inflammation induced by the coil materials (13), vasospasm and subarachnoid hemorrhage and blood pressure changes after surgery of ruptured arterial aneurysms. Even without specific risk factors, causative factors for CIE include hyperosmolality, neurotoxicity and the vascular toxicity of contrast media.

In reports published to date, the onset of CIE often occurred within a few hours to a few days after coil emboliza- tion or cerebral angiography. In contrast, our patient developed symptoms approximately 30 days after coil embolization. In three previously reported cases of subacute onset, CIE occurred at approximately one month and three weeks post-procedure, respectively $(13,17)$. The present patient showed only very mild symptoms of agraphia and right upper extremity hemiparesis and may not have noticed symptoms for some time during daily activities.

Typical radiological findings of CIE include abnormal cortical contrast enhancement and edema, subarachnoid contrast enhancement and striatal contrast enhancement (16). Abnormal findings on brain MRI were identified in 16 of the previously reported cases $(3,6,7,9,11-18)$. Because abnormal findings on brain MRI are consistent with vasogenic edema, CIE has recently been occasionally included in the category of posterior reversible encephalopathy syndrome (PRES). In previous reports, the prognosis has typically been good, with the exception of one case (16). As observed in those with PRES, clinical sequelae are absent and imaging shows reversible findings in most patients.

A large amount $(316 \mathrm{~mL})$ of non-ionic contrast agent was used in the current case. CIE reportedly occurs when great volumes of iodinated contrast are employed (13), while the amount of contrast agent used in the above cases varied from $12 \mathrm{~mL}$ to $300 \mathrm{~mL}$ (mean, $125 \mathrm{~mL}$ ). Therefore, no clear correlation exists between the incidence of CIE and the amount of contrast agent.

In the present case, steroid pulse therapy was administered for three days with $1,000 \mathrm{mg}$ of methylprednisolone in 
Table. Cases of CIE after Cerebral Angiography with CT- or MRI-confirmed Lesions

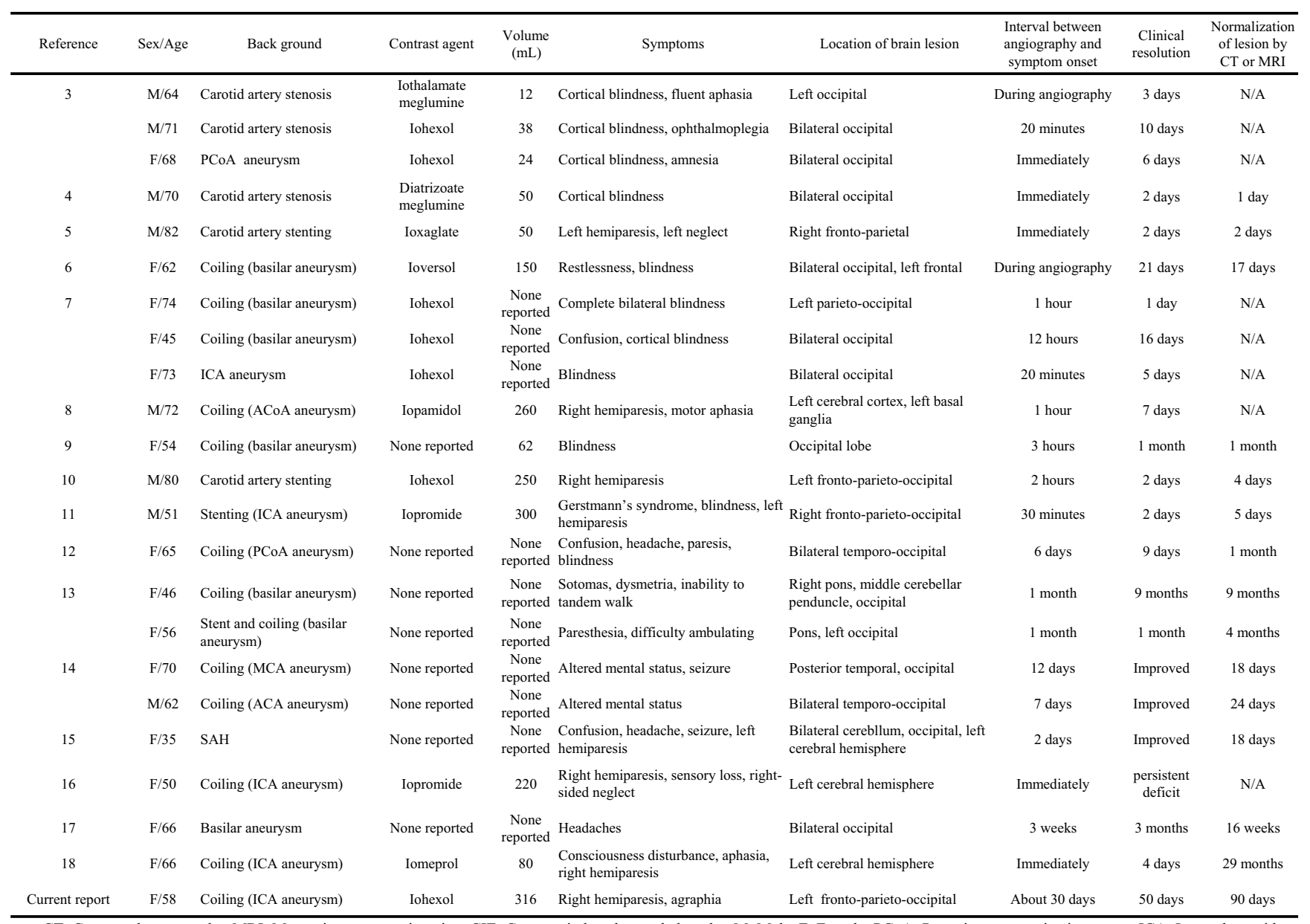

CT: Computed tomography, MRI: Magnetic resonance imaging, CIE: Contrast-induced encephalopathy, M: Male, F: Female, PCoA: Posterior communicating artery, ICA: Internal carotid artery, ACoA: Anterior communicating artery, MCA: Middle cerebral artery, ACA: Anterior cerebral artery, SAH: Subarachnoid hemorrhage, N/A: Not applicable

order to reduce the patient's edema. Her symptoms improved after treatment, signifying either steroid responsiveness or spontaneous resolution. If we assume steroid responsiveness, then the presence of immune-mediated encephalopathy may also be considered; however, no immediate adverse reactions, such as rashes or nausea, were noted following contrast injection, no eosinophilia was evident at the time of hospital admission or during follow-up and DLST to iohexol yielded negative results. An allergic inflammatory reaction thus seems unlikely.

In conclusion, endovascular coil embolization for unruptured arterial aneurysms is a widely performed neurosurgical intervention. Therefore, the possibility of complications, such as CIE, should be kept in mind in cases involving postoperative abnormal neurological findings, even during the subacute period.

\section{The authors state that they have no Conflict of Interest (COI).}

\section{References}

1. Fischer-Williams M, Gottschalk PG, Browell JN. Transient cortical blindness. An unusual complication of coronary angiography. Neurology 20: 353-355, 1970.
2. Potsi S, Chourmouzi D, Moumtzouoglou A, Nikiforaki A, Gkouvas K, Drevelegas A. Transient contrast encephalopathy after carotid angiography mimicking diffuse subarachnoid haemorrhage. Neurol Sci 33: 445-448, 2012.

3. Lantos G. Cortical blindness due to osmotic disruption of the blood-brain barrier by angiographic contrast material: CT and MRI studies. Neurology 39: 567, 1989.

4. Shyn PB, Bell KA. Transient cortical blindness following cerebral angiography. J La State Med Soc 141: 35-37, 1989.

5. Dangas G, Monsein LH, Laureno R, et al. Transient contrast encephalopathy after carotid artery stenting. J Endovasc Ther 8: 111113, 2001.

6. Shinoda J, Ajimi Y, Yamada M, Onozuka S. Cortical blindness during coil embolization of an unruptured intracranial aneurysm: case report. Neurol Med Chir (Tokyo) 44: 416-419, 2004.

7. Saigal G, Bhatia R, Bhatia S, Wakhloc AK. MR findings of cortical blindness following cerebral angiography: is this entity related to posterior reversible leukoencephalopathy. AJNR Am J Neuroradiol 25: 252-256, 2004.

8. Uchiyama Y, Abe T, Hirohata M, et al. Blood brain-barrier disruption of nonionic iodinated contrast medium following coil embolization of a ruptured intracerebral aneurysm. AJNR Am J Neuroradiol 25: 1783-1786, 2004.

9. Niimi Y, Kupersmith MJ, Ahmad S, Song J, Berenstein A. Cortical blindness, transient and otherwise, associated with detachable coil embolization of intracranial aneurysms. AJNR Am J Neuroradiol 29: 603-607, 2008.

10. Fang HY, Kuo YL, Wu CJ. Transient contrast encephalopathy af- 
ter carotid artery stenting mimicking diffuse subarachnoid hemorrhage: a case report. Catheter Cardiovasc Interv 73: 123-126, 2009.

11. Guimaraens L, Vivas E, Fonnegra A, et al. Transient encephalopathy from angiographic contrast: a rare complication in neurointerventional procedures. Cardiovasc Intervent Radiol 33: 383-388, 2010.

12. Jang HW, Lee HJ. Posterior reversible leukoencephalopathy due to "triple H" therapy. J Clin Neurosci 17: 1059-1061, 2010.

13. Skolarus LE, Gemmete JJ, Braley T, Morgenstern LB, Pandey A. Abnormal white matter changes after cerebral aneurysm treatment with polyglycolic-polylactic acid coils. World Neurosurg 74: 640644, 2010.

14. Giraldo EA, Fugate JE, Rabinstein AA, Lanzino G, Wijdicks EFM. Posterior reversible encephalopathy syndrome associated with hemodynamic augmentation in aneurysmal subarachnoid hemorrhage. Neurocrit Care 14: 427-432, 2011.
15. Voetsch B, Tarlov N, Nguyen TN, et al. Asymmetric posterior reversible encephalopathy syndrome complicating hemodynamic augmentation for subarachnoid hemorrhage-associated cerebral vasospasm. Neurocrit Care 15: 542-546, 2011.

16. Leong S, Fanting NF. Persistent neurological deficit from iodinated contrast encephalopathy following intracranial aneurysm coiling. A case report and review of the literature. Interv Neuroradiol 18: 33-41, 2012.

17. Naito H, Yamazaki Y, Takahashi T, Ochi K, Kiura Y, Matsumoto M. A case of posterior reversible encephalopathy syndrome associated with coil embolization of a basilar apex aneurysm. Rinsho Shinkeigaku (Clin Neurol) 53: 518-525, 2013 (in Japanese, Abstract in English).

18. Kubota T, Yamazaki T, Maeda M, et al. A case of contrast-induced encephalopathy after coil embolization of unruptured internal carotid artery aneurysm. Nosotchu no Geka (Surg Cereb Stroke) 41: 116-122, 2013 (in Japanese, Abstract in English).

(C) 2014 The Japanese Society of Internal Medicine http://www.naika.or.jp/imonline/index.html 